\title{
MARESye: A hybrid imaging system for underwater robotic applications
}

\author{
Andry Maykol Pinto ${ }^{1, *}$, Anibal C. Matos \\ INESC Technology and Science, Faculty of Engineering, University of Porto, Rua Dr. Roberto Frias, Porto $s / n$ 4200-465, Portugal
}

\section{A R T I C L E I N F O}

\section{Keywords:}

Underwater imaging

Image fusion

Active imaging

Passive imaging

Underwater

Robotics

\begin{abstract}
A B S T R A $\mathrm{T}$
This article presents an innovative hybrid imaging system that provides dense and accurate 3D information from harsh underwater environments. The proposed system is called MARESye and captures the advantages of both active and passive imaging methods: multiple light stripe range (LSR) and a photometric stereo (PS) technique, respectively. This hybrid approach fuses information from these techniques through a data-driven formulation to extend the measurement range and to produce high density 3D estimations in dynamic underwater environments. This hybrid system is driven by a gating timing approach to reduce the impact of several photometric issues related to the underwater environments such as, diffuse reflection, water turbidity and non-uniform illumination. Moreover, MARESye synchronizes and matches the acquisition of images with sub-sea phenomena which leads to clear pictures (with a high signal-to-noise ratio). Results conducted in realistic environments showed that MARESye is able to provide reliable, high density and accurate 3D data. Moreover, the experiments demonstrated that the performance of MARESye is less affected by sub-sea conditions since the SSIM index was 0.655 in high turbidity waters. Conventional imaging techniques obtained 0.328 in similar testing conditions. Therefore, the proposed system represents a valuable contribution for the inspection of maritime structures as well as for the navigation procedures of autonomous underwater vehicles during close range operations.
\end{abstract}

\section{Introduction}

Nowa days, the number of autonomous underwater robots (AUV) that resort to imaging systems is increasing worldwide [34], a trend that is being supported by several economical sectors related to fisheries, environmental monitoring, inspection of offshore wind farms and the oil\&gas industry. These sectors are currently demanding for underwater imaging solutions with the ability to provide reliable information during tasks related with 3D mapping and object manipulation. Moreover, imaging solutions can produce relevant data for the navigation of medium-sized AUVs (autonomous underwater vehicles) or ROVs (remotely-operated vehicles). Although underwater imaging systems have the potential to improve the perceptual capability of AUV/ROV, existing visual solutions are strongly affected by photometric limitations related to the intrinsic nature of the sub-sea environment: poor visibility, the presence of suspended particles, light absorption and backscattering [36]. The majority of underwater robotic applications with visual perception capabilities usually resort to a single high resolution camera in a dome port or to off-the-shelf stereoscopic systems in watertight enclosures. Obviously that harsh underwater environments cause severe photometric issues that alter the performance of conventional sensors (such as, the BumblebeeV2 and the MobileRanger), affecting the accuracy and precision of the $3 \mathrm{D}$ estimations.

This research presents a reliable imaging principle for acquiring the 3D perceptual information in underwater environments. An imaging solution called MARESye is proposed which combines three key components ${ }^{2}$ : (1) a hybrid-based approach; (2) a range-gated imaging scheme; and, (3) a data-driven formulation. A brief introduction of the proposed system is provided in this research which includes an overview of the system architecture, a description of several features that enhance the visual capacity of the system and a set of results that demonstrate the ability of MARESye to operate in underwater scenarios.

Therefore, the contributions of this research include:

1. A new representation for underwater imaging systems with a strong potential to enhance the remote sensing capability of AUV and ROVs. This representation retrieves textured and 3D information from underwater scene with a high degree of robustness, precision and accuracy.

\footnotetext{
2 The technology presented in this research is protected by a patent application EP 18169993.5 and PT110671 (patent pending).
}

\footnotetext{
* Corresponding author.

E-mail address: andry.pinto@fe.up.pt (A.M. Pinto).

${ }^{1}$ Departamento de Electrotecnia da Faculdade de Engenharia da Universidade do Porto, Rua Dr. Roberto Frias, s/n 4200-465 Porto, Portugal
} 
2. A highly flexible architecture for 3D perception that fuses active and passive imaging techniques: multiple Light Stripe Range (LSR) and Photometric Stereo (PS) techniques, respectively.

3. A study about the advantages of combining a hybrid approach with a range-gated acquisition scheme. A data-driven formulation is used to aggregate different sources of visual data with a higher signal-tonoise ratio (SNR).

4. A novel optical system called MARESye. This system lays down on several imaging principles to increase its robustness to some photometric issues induced by the underwater environment. Overall, data obtained by MARESye is less affected by the backscatter component and motion blur.

5. Qualitative and quantitative evaluations considering underwater scenarios with different testing conditions. The current research goes one step further by presenting a visual-based approach that meets the visual requirements of underwater robots for specialized tasks such as, the inspection and monitoring of man-made structures.

The article is organized as follows: Section 2 presents a brief review of visual solutions and methods used by underwater robotic systems to acquire 3D information. Afterwards, Section 3 presents the MARESye system and its experimental evaluation is demonstrated in Section 4. Several experiments were conducted in a realistic underwater scenario subjected to different testing conditions. Moreover, the results include many comparisons of the proposed system with a conventional visual solution. Experimental considerations show that MARESye performs satisfactorily better and, therefore, the combination of active and passive imaging techniques using a data-driven formulation proposed in this research represents a clear and reliable alternative to conventional visualbased solutions. Finally, Section 5 clarifies the most important conclusions of this research.

\section{Related work}

Recent developments in the field of visual perception have contributed to the appearance of new and sophisticated autonomous robots and applications $[30,35]$. Data from multiple sensors of different types is combined in the majority of these applications to produce a better interpretation and more reliable inference than single source data [18].

Acoustic sensors are often used by underwater applications to conduct bathymetric surveys, obstacle avoidance and long range navigation [21] however, they have severe limitations related with the cost and the inability to provide texture information and low sampling frequencies. On the contrary, optical systems can provide dense and accurate texture information updated at high speed, which is crucial for tasks such as, underwater manipulation, oceanographic studies and marine biology, shipwreck surveys and object identification. Although optical systems can increase the feasibility of missions can be carried out by AUVs [10], they still need to overcome the photometric limitations of sub-sea conditions namely, non-uniform lighting and colour filtering, poor visibility due to light attenuation and scattering which is caused by suspended particles in the medium or the abundance of marine life [1,7].

Generic and conventional systems for 3D reconstruction are usually classified into passive or active imaging techniques.

Underwater passive imaging uses ambient sunlight at shallow waters or light sources (i.e., spotlights and LEDs) at deeper waters just to ensure the sufficient lighting of the scenario. This category is based on photogrammetry where the underwater scene is captured from different viewpoints to retrieve 3D information. A stereoscopic system estimates the stereo correspondence from images obtained by different viewpoints for close and medium range measurements. Oleari et al. [29] demonstrates a stereoscopic setup formed by two industrial cameras that were developed within the MARIS project. The system estimates a point cloud at 12.5 frames per second $(1292 \times 964$ resolution) using SAD (Standard Absolute Differences correlation method) with an acceptable quality. The system does not provide additional imaging methodologies to accommodate the photometric limitations of underwater environments and, therefore, the authors did not discuss the robustness and reliability of the 3D acquisition. In a recent work [38], the previous system was integrated in a ROV to provide visual information for object manipulation. Experiments conducted in a water pool with different light conditions (sunny, cloudy and night with vehicle lights) but without turbidity constraints have demonstrated the challenges of perceiving the underwater environment, in particular, the setup between the illumination of scene and the camera shutter. In recent biological studies, the use of stereo systems are becoming increasingly popular to identify individual fishes and to characterize the biological behavior of underwater species [19]. The research [42] presents a stereo system called TrigCam. The system illuminates the scene with a red light to minimize the effect of light during the biological activity of fishes, whose detection will trigger the acquisition of a high-resolution image pair under white illumination. The research work found in [4] proposes a stereo graph-slam method, where a SPARUS II has a conventional stereo sensor (a Bumblebee with a watertight enclosure). Results do not capture all phenomena related with sub-sea conditions since they were obtained in a simulated scenario however, they have demonstrated that this method has the potential to reduce the trajectory error of the underwater vehicle. A more advanced vision system [2] formed by two stereoscopic cameras (Bumblebee) enhances the maneuverability and manipulation capability of underwater robots. This system is called Fugu-f, has a depth rate of $250 \mathrm{~m}$ and can be adaptable to different vehicles. This is one of the most advanced underwater imaging systems available in the scientific community since it was evaluated in different robotic platforms: Girona500 [37] (from CIRS - Centre for Research in Underwater Robotics-University of Girona) and Nessie VI from Heriot-Watt University [27]; and, evaluated in both water tanks and real sea scenarios (within the TRIDENT project). The results demonstrated that this system can be used for different tasks (such as, object detection and grasping) however, a quantitative analysis of the accuracy of Fugu-f for 3D mapping is missing (instead, the authors resorted to several visual-guided operations to justify the performance of Fugu-f). Madjidi and Negahdaripour [26] proposed a multi-scale implementation to estimate dense optical flow fields from a stereoscopic sensor that can be used to determine a dense stereo disparity field.

Underwater active imaging uses the projected pattern of light to determine the distance between the device and the illuminated target by means of time-of-flight or triangulation principle. The international community has been interested on underwater active sensors [16] which are typically employed for long and medium range acquisitions. Moreover, they are able to enhance the quality of images perceived by the receiver by controlling a light pattern. An underwater active stereo system can also be used to capture dynamic objects in water such as, swimming fishes like in [17]. This work studies the refraction caused by waterproof housings and proposes a projector-camera calibration approach to define the refraction model of flat housings. A laser-based range sensor for underwater environments formed by one camera and two lasers projecting continuous vertical lines is presented in [13]. The authors have reported a calibration error of $5 \%$ and results obtained in a controlled tested scenario (with very clear water) showed a set of measurements with a drift close to $0.04 \mathrm{~m}$. The work presented in Bruno et al. [3] concludes that the structured light can be used in underwater environments for a 3D reconstruction in low turbidity waters. The solution presented in that research requires a camera, projector and a physical structure capable of protecting the entire system when it is submerged in water. The presence of a projector in this kind of applications makes it possible to conduct range measurements with high quality because of the welldefined pattern that is normally projected in the scene is well defined however, the electric power required by this device is non-negligible which reduces the autonomy of a robotic vehicle. The work of Amin and Brian [39], extends a structured light technique based on a camera and a projector fixed above water. Thus, the scene is illuminated with spatially varying intensity patterns to allow the extraction of the 3D surface of objects. Despite of its accuracy, this technique can only be 
used in specific case-scenarios where the observer does not move. Advances in remote visual sensor technology have been followed closely by advances in the artificial illumination.

More recently, the image fusion has received significant attention and became a relevant research field with many systems [20] and algorithms $[8,12,23,24,45]$ presented in the last years. The limited visual perception capability of AUVs restricts the use of such vehicles to medium/long range missions however, researchers are developing new techniques to enhance the visibility of underwater optical systems $[22,25]$.

Therefore, the development of visual methods for perceiving elements below water is crucial and, in this context, the present research work proposes a novel underwater imaging system called MARESye: a hybrid imaging system that can be easily installed in different underwater robotic applications such as, AUV and ROVs. The advantage of MARESye when compared to conventional visual sensors relies on the fact that the proposed system conducts temporal fusion over multiple imaging acquisition principles to reduce the photometric limitations that usually affect imaging solutions operating in underwater scenes.

\section{MARESye - Novel insights for underwater imaging}

A novel imaging system called MARESye is proposed in this article. This system resorts to a hybrid imaging formulation that makes it possible to perceive 3D structure of underwater scenarios. This section aims to introduce the challenges of operating imaging systems in underwater scenarios, as well as, to describe the MARESye system in terms of its concept and main architecture.

\subsection{The challenges of underwater imaging}

The light interacts with the water medium through two phenomena: absorption (loss of power which depends on the index of refraction of the medium) and scattering (deflection from a straight-line propagation path). Thus, the irradiance $E(r)$ at position $r$ can be described as a function of the absorption and scattering coefficients of the medium. ${ }^{3}$ Under these terms, a simple analysis can be conducted by considering the Lambert-Beer empirical law along with the assumption of a homogeneous and isotropic water medium [40]. Eq. (1) demonstrates how both components (scattering and absorption) decrease the contrast and attenuate the light intensity of images.

$E(r)=E(0) e^{-a r} e^{-b r}$

where $a$ and $b$ are the absorption and scattering coefficients. Considering the image formation model of Jaffe [15], an underwater image can be expressed as the linear superposition of the direct component $\left(E_{d}\right)$, the forward-scattered component $\left(E_{f}\right)$ and the backscatter component $\left(E_{b}\right)$, see Eq. (2).

$E_{T}=E_{d}+E_{f}+E_{b}$,

where $E_{T}$ is the total irradiance. The $E_{b}$ represents the light that does not contain information about the object since it was reflected by the medium. The $E_{b}$ adds a noise component and reduces the image contrast [6]. The $E_{f}$ represents the amount of light reflected by the object which is forward scattered at small angles and its practical evidence is an image blur. The majority of research works available in the literature are usually focused on proposing post-processing methods to mitigate $E_{f}$ and $E_{b}$ components. This research work takes a different path by incorporating a set of imaging principles directly into an optical system. These innovations aim to increase the robustness of the image formation by minimizing the influence of $E_{b}$ (and $E_{f}$ ).

\footnotetext{
${ }^{3}$ More information can be found in [40].
}
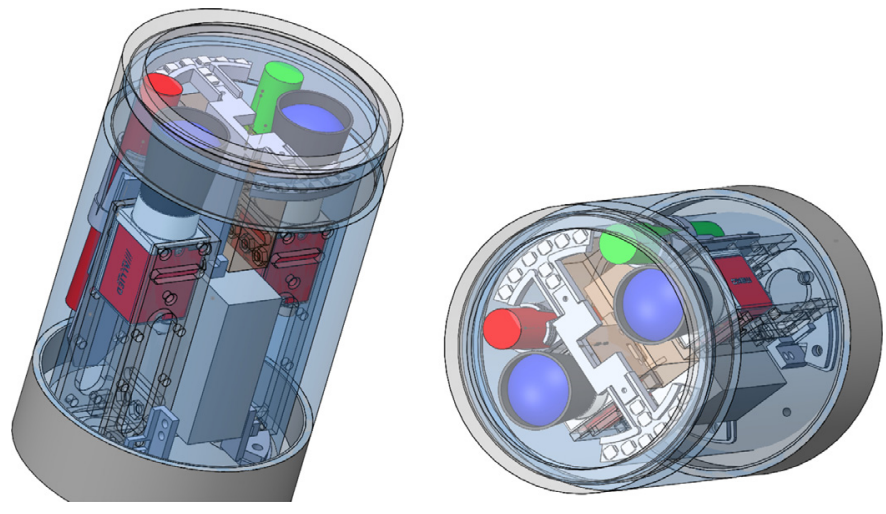

Fig. 1. The MARESye concept design. The PS system is formed by two cameras and the LSR system is formed by a set of 2 lasers and two cameras.

\subsection{The optical system}

The MARESye is an advanced optical system that combines a hybridbased visual approach controlled by a range-gated imaging scheme with a data-driven formulation. The internal architecture of MARESye is formed by three main modules: multiple Light Stripe Range (LSR), Photometric Stereo (PS) and the Range-Gated Synchronizer. The 3D information received from the LSR and the PS modules are combined and filtered in a fourth module named, 3D fitting and Texture (which is out of the scope of this research). The hardware of MARESye is formed by two cameras (left and right), two laser stripes (red and green wavelengths), a triggering system, and the illumination module (high intensity LEDs). Fig. 1 presents different viewpoints for the concept design of MARESye, in particular, the physical layout of the entire system. These modules are organized in a functional structure, see Fig. 2. All components are interconnected with software modules that extract the 3D information from visual data. The architecture implemented in MARESye is depicted in Fig. 2. Outputs of the system comprise a pair of images from the PS, point clouds retrieved by each 3D imaging technique (4 pointclouds from the LSR and a dense point cloud from the PS). These point clouds can be combined further by applying 3D filtering techniques [28]. This architecture will be discussed with details during this section.

\subsubsection{The hybrid imaging approach}

The hybrid imaging approach proposed by MARESye brings together active and passive imaging principles: multiple Light Stripe Range (LSR) and a Photometric Stereo (PS) technique. Fig. 3 presents the physical implementation of MARESye when operating in total darkness.

The LSR is an active technique that comprises a set of stripes of light generated by laser diodes that are projected in the scene. The 3D information is recovered from these laser stripes by means of triangulation, see Algorithm 1. Laser technology has a strong advantage in underwater scenes since emitters concentrate an intense light over a very narrow area which extends the propagation distance of the light. The segmentation of the laser stripe is conducted in the $\mathrm{YCrCb}$ because this space is more photometric invariant to light changes and the chroma components of this space are less intercorrelated $[28,34]$. Then, a segmentation procedure takes into consideration the chroma values $(\mathrm{Cr}$ and $\mathrm{Cb}$ ) and confidence intervals. Afterwards, the segmentation results are redefined by a watershed approach (eg., see the description presented in $[11,31,32])$ that groups pixels with similar spatial position and color. Morphological operators increase the spatial connectivity of the segmentation result.

Before extracting the 3D information, a calibration must be performed to estimate the spatial arrangement of each laser according to the camera frame. Thus, the triangulation determines the three-dimensional points by intercepting $2 \mathrm{D}$ points from the segmentation with the plane 


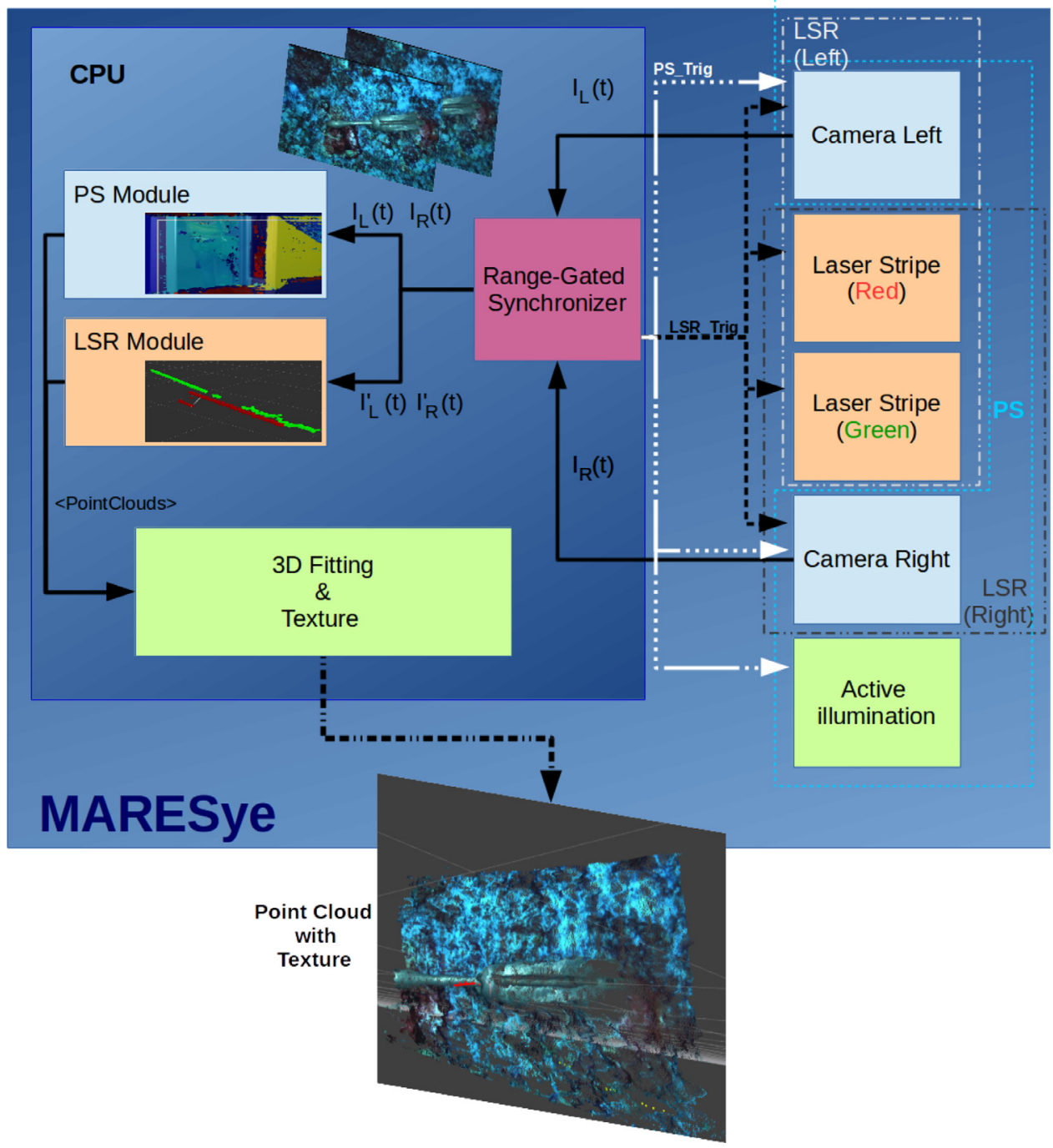

Fig. 2. Depicts the interaction scheme between different modules that comprise the MARESye. The internal architecture of this system is formed by hardware and software components that were designed to enhance the visual perception capability of mobile robots operating in underwater scenarios.

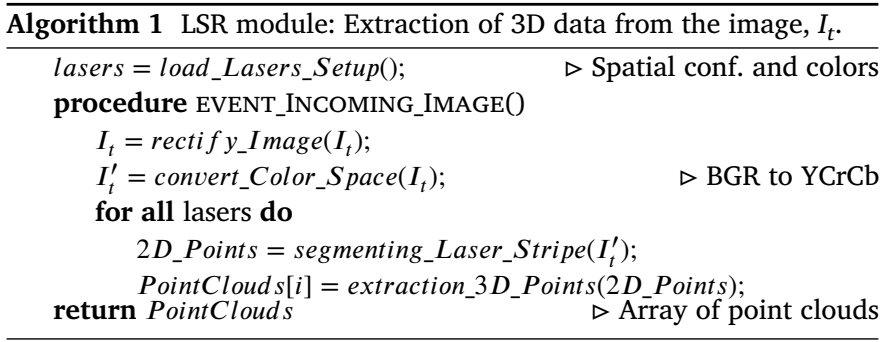

equations available (the camera matrix is known), see Eqs. (3)-(5)

$Z=-\frac{(a \times x+b \times y+d)}{c}$

$X=Z \times x$

$Y=Z \times y$

where $x$ and $y$ are the 2D points described in the image plane and, the $a, b, c$ and $d$ define a plane that characterizes the laser-camera setup. The $X, Y$ and $Z$ are 3D coordinates of a point represented in the camera frame. This triangulation process is repeated for each pair camera-laser.

An example of this process can be seen in Fig. 4. The biggest advantage of an active imaging approach is the high accuracy and robustness of data that can be retrieved from the scene. Usually, the propagation of light in water is seriously compromised by the scatter and absorption of light caused by the medium which reduce the image contrast and intensity [1]. The LSR is able to calculate object sizes with a high degree of precision because it minimizes the backscatter influence and increases the image contrast.

The PS is a passive technique that reconstructs the 3D surfaces based on different viewpoints. It provides point clouds having a higher density of 3D data when compared to LSR. The knowledge of the relative position and orientation of both cameras (separated by a rigid baseline) makes possible to the extract of the three-dimensional surface by applying the stereoscopy principle: 3D points are generated by 2D point pairs, see Fig. 5. Conventional stereoscopy can be performed in two distinct ways namely, sparse (a set of point pairs) or dense (for every pixel). Both ways can be used in the MARESye system, depending on the application. A key element of the PS module is the estimation of the disparity of images acquired in different viewpoints. This is often called as the correspondence problem (the challenge of associating 2D point pairs). The perception of depth arises from disparity by means of triangulation (depth is inversely proportional to disparity). The set of 3D points (also known as a point cloud) can be more or less dense depending on the amount of valid stereo correspondences. Recent correlation techniques have been proposed by the scientific community to solve this correspondence problem $[9,43,44]$, with distinct results in terms of depth accuracy, computation speed and noise. In practice, the PS module is suitable 


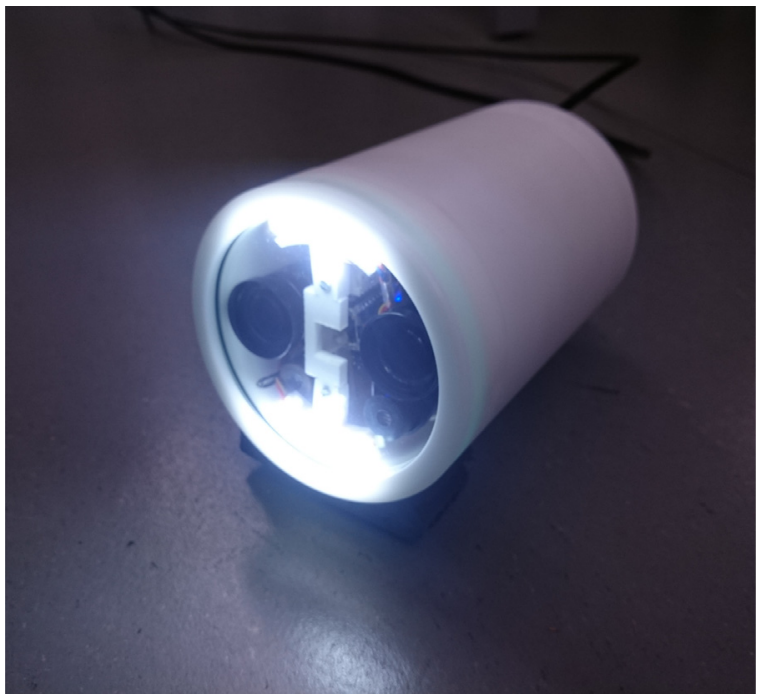

Fig. 3. Depicts the MARESye prototype operating in total darkness. This figure captures the instant of time when the PS (Photometric Stereo) module is grabbing frames (estimating a dense point cloud).

for environments having dynamic elements because it provides data that is richer in terms of texture and 3D information.

The Algorithm 2 presents high-level procedures for extracting 3D information based on a stereo rig. The implementation is straightforward in computer vision: the calibration of the stereo rig should is conducted in aerial and underwater scenarios, the disparity estimation follows [14], and 2D points with no disparity must be removed.

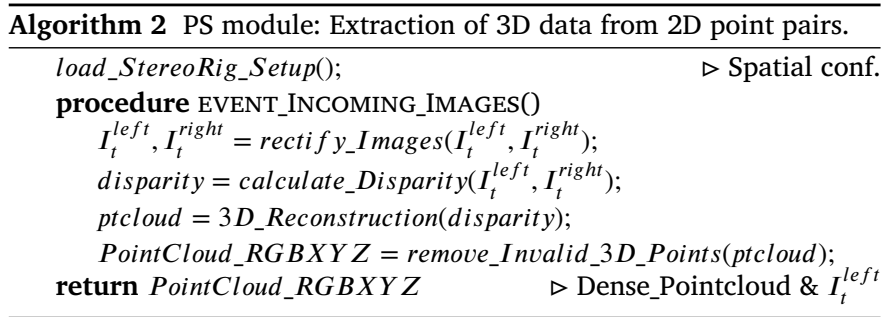

\subsubsection{The range-gated imaging scheme}

The MARESye has the ability to control the image formation process in both cameras (e.g., the starting instant and duration). The range-gated imaging concept lays on the principle that the reflected light source pulsed from the LEDs or lasers is accumulated by an optical sensor, over a specified time and the camera shutter only opens after a short period of time. Thus, the camera sensor receives the light reflected from the object and blocks the backscattered photons. The exposer time selects the depth range where the light reflected is perceived at the sensor location. This approach is one of the most effective solution for visual perception in turbid waters [6] since it increases the image contrast by reducing the backscattering component.

Fig. 6 illustrates the how the backscattering component affects the underwater imaging sensors: a fraction of light that passes through water is scattered back to the camera before reaching the target. Conventional optical systems are severely affected by $E_{b}$ which is mainly caused by particles suspended along the water column. These particles increase the water turbidity and reduce both the visibility and the measurement range of optical systems. In this context, the range-gated feature available on MARESye delays the image acquisition by a short period of time after the scene be illuminated by a light source: the artificial illumination module or the pulsed light from the LSR. An active-illumination functionality is implemented based on high intensity LEDs (light emit-

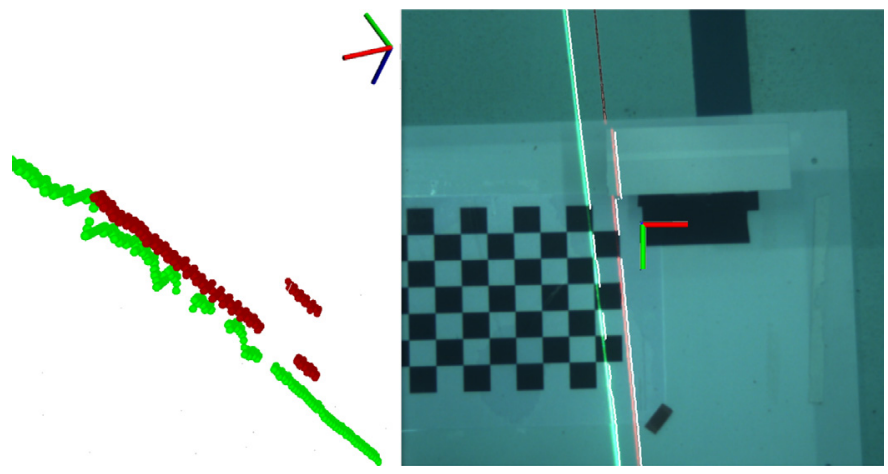

Fig. 4. The extraction of 3D information (on the left) from the underwater image (on the right), considering the Algorithm 1 . The 3D object coordinate ( $X, Y$, $Z$ ) is represented in the upper-right corner and the 2D image plane coordinate $(x, y)$ is represented on the center of the first and second image, respectively. The trial captures a step function with a real amplitude of $0.08 \mathrm{~m}$ and the LSR module measured 0.083 (represented with a spatial resolution of $0.004 \mathrm{~m}$ ).

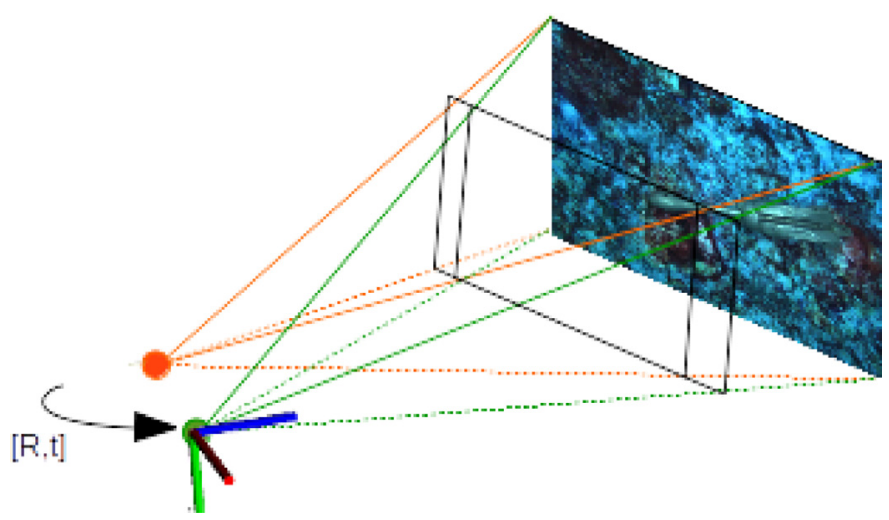

Fig. 5. The three-dimensional surface can be estimated by overlapping areas of both two images (orange and green) and by knowing the relative pose between both observations ( $R$ and $t$ are the rotation and translation, respectively) as well as the calibration parameters of cameras. The $3 \mathrm{D}$ object coordinate $(X, Y, Z)$ is depicted for the right camera. (For interpretation of the references to colour in this figure legend, the reader is referred to the web version of this article.)

ting diodes) to create a close-to-isotropic light source that illuminates the underwater scenario when the system is operating in total darkness.

Fig. 2 shows that MARESye generates several triggering signals $\left(P S_{\text {Trig }}\right.$ and $L S R_{\text {Trig }}$ ) that are sent to cameras with external triggering: the $P S_{\text {Trig }}$ activates the artificial illumination and the PS modules, and the signal $L S R_{\text {Trig }}$ activates the LSR module. These periodic signals are generated by an external trigger control unit called Range-Gated Synchronizer whose main objective is to ensure that images are acquired in the correct instant of time. The signals $P S_{\text {Trig }}$ and $L S R_{\text {Trig }}$ are sent to the cameras after a delay period $T_{\text {start }}$ of these signals were received by the PS and LSR units (this subject is discussed in the next section).

In this context, the camera shutter waits for the time that light takes to propagate from the emitter to the target. Ideally, $T_{\text {start }}$ is controlled according to that distance and, the exposure time should be adjusted relatively to the $T_{\text {stop }}-T_{\text {start }} \cdot{ }^{4}$ The goal of this approach is to reduce the amount of backscattering component that is perceived by the optical sensors which is mainly caused by suspended particles close to the observer, see Fig. 6. Therefore, MARESye incorporates a range-gated feature that synchronizes the acquisition of image with sub-sea phenomena. The gated timing approach limits the exposure time of the optical

4 The exposure time is fixed in current version of MARESye however, it will be dynamically adjusted in future versions. 


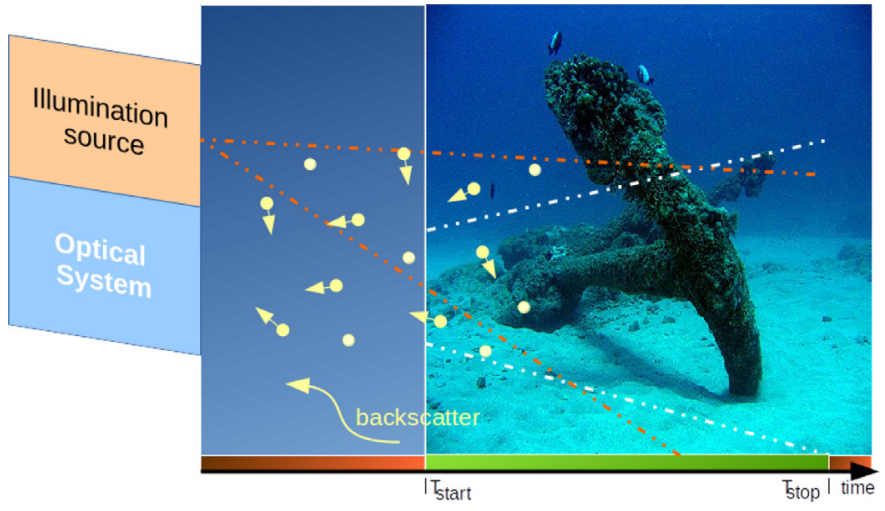

Fig. 6. Depicts the effect of range-gated optical systems in underwater scenarios. The illumination source could be the artificial illumination module and the lasers lights from the LSR module, see Fig. 2.

system to half of the travel time of the light pulse (laser or LED) scattered by the target at a defined distance. A practical advantage of this range-gated approach relates with the robustness of the image forma- tion regarding the influence caused by other sources of light or external interferences (exposure time versus the diaphragm's aperture) [33]. Although the influence of $E_{b}$ can be reduced by a range-gated approach (synchronization of the light source and camera), the $E_{f}$ cannot be quantitatively reduced by this method since this component reaches the image detector approximately at the same time than $E_{d}[6]$.

\subsubsection{A data-driven formulation}

The data-driven formulation proposed in this research follows a time discrimination pattern to aggregate the 3D information generated by the LSR and PS approaches. In this way, each image captures the light returned from a single light pulse that can be driven by the LSR or by the PS module. In practice it means that, the optical components of MARESye are sequentially gated by the LSR (without the activeillumination source) and by the PS (with the active-illumination source) to minimize the amount of backscattering light that reaches the optical sensors, as well as, to mitigate the motion blur that arrives when the system is installed on the AUV or ROV.

A data-driven scheme synchronizes the acquisition instant of multiple cameras based on distinct time events. These time events are represented by signals $P S_{\text {Trig }}$ and $L S R_{\text {Trig }}$ which control the camera exposure (orange) delayed by $T_{\text {start }} / 2$, see Fig. 7 (a). Signals describing the ON

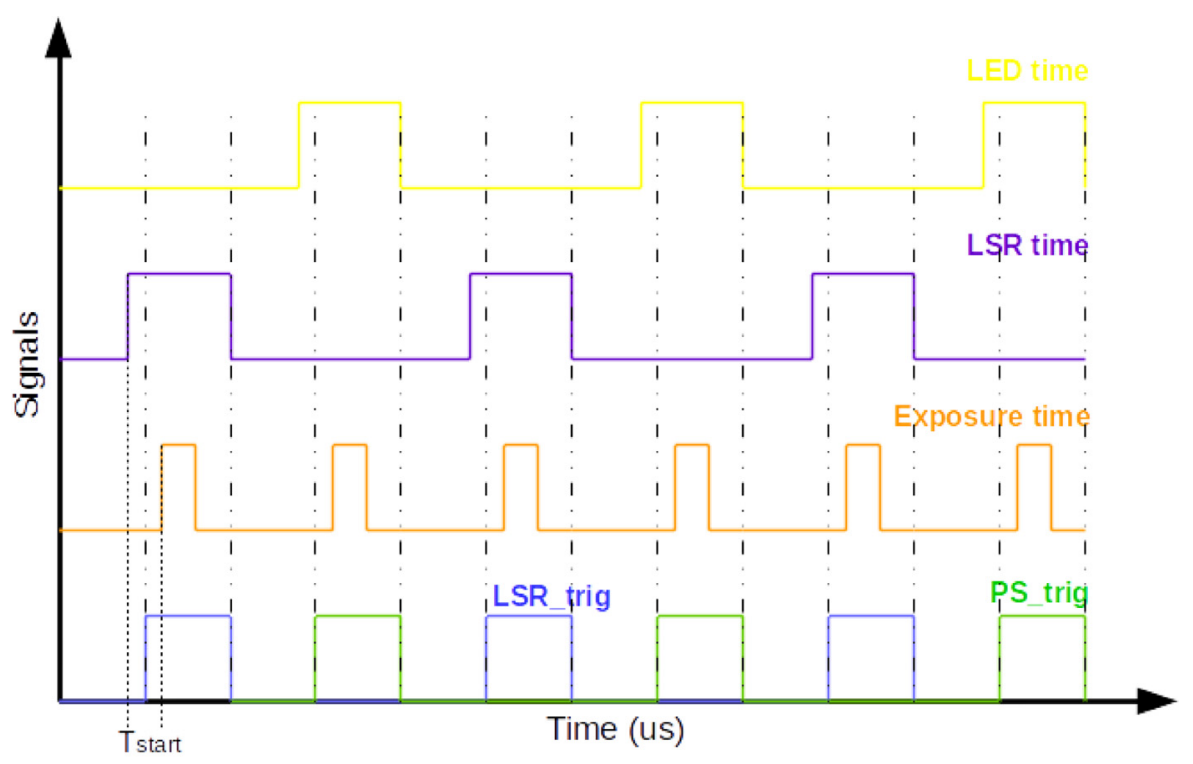

(a)

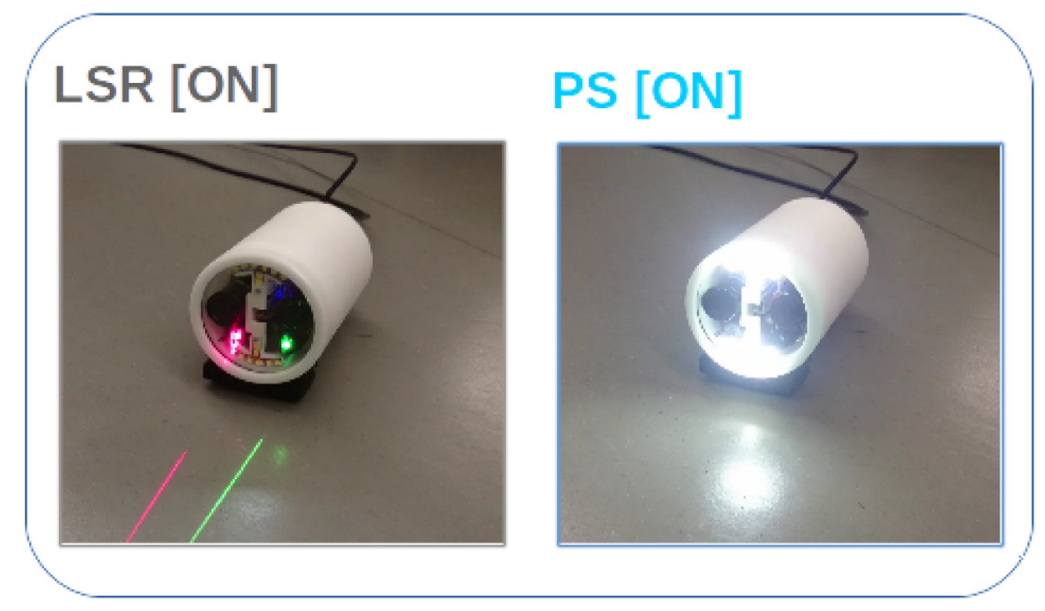

(b)
Fig. 7. A data-driven scheme creates two (or more) operating modes for MARESye. Picture on the left depicts the LSR_ON mode (where MARESye is using structured light from the LSR module to extract 3D data). Picture on the right depicts the PS_ON mode (where MARESye illuminates the scene and grabs frames that are used to extract 3D data based on the PS module). 


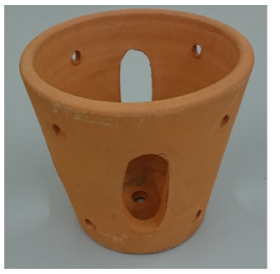

(a)

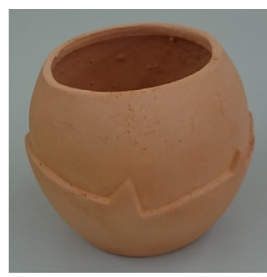

(b)

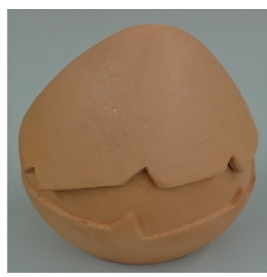

(c)

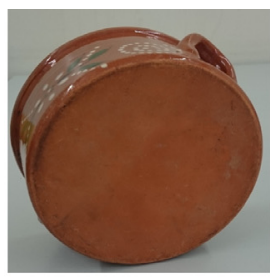

(d)
Fig. 8. Objects used in laboratory tests. V-object in Fig. 8(a), OS-object in Fig. 8(b), OC-object in Fig. 8(c) and T-object in Fig. $8(\mathrm{c})$ have a height of $0.16 \mathrm{~m}, 0.13 \mathrm{~m}$, $0.19 \mathrm{~m}$ and $0.14 \mathrm{~m}$, respectively. period of the active-illumination module or lasers are represented by the LED time (on yellow) and the LSR time (on purple) curve, respectively. These signals anticipate (by $T_{\text {start }} / 2$ ) the time events: LSR_ON and PS_ON. In this way, the light sources (LSR or active-illumination) remain $\mathrm{ON}$ during the exposure period of the multiple cameras which increases the accuracy (real-time) of the synchronization process, reduces the power consumption and enables high speed acquisitions [33].

Therefore, MARESye follows a time discrimination pattern to create two (or more) operating modes ${ }^{5}$ : LSR_ON and PS_ON, see Fig. 7(b). Optical sensors are able to capture the laser and LED light in distinct time slots. The PS_ON mode (right picture from Fig. 7(b)) shows that MARESye acquires images that are analyzed by the PS module: estimates the 3D surface of the scenario illuminated by the active-illumination module. Overall, this module artificially illuminates the scene based on a rapidly variation of light pulses (strobe) which has several advantages: it is more energy-efficient than continuously emitted light sources and reduces the motion blur. This solution is, therefore, suitable for autonomous vehicles. The LSR_ON mode (left picture from Fig. 7(b)) recovers the three-dimensional structure of the environment from multiple laser-camera triangulations. In LSR mode, the active-illumination module remains OFF to decrease the amount of external light interferences during the segmentation of the laser stripes. Dalgleish et al. [5] demonstrated that pulse-gated laser sources provide a much better performance for underwater imaging than continuously excited lasers. Several studies defend the use of laser-based triangulations sensors due to its narrow spectral width and high peak power. Many authors consider that pulse-gated laser sources is the optimal technology for extended range measurements in underwater scenes $[5,6]$.

In practice, MARESye combines multiple laser-based range-gated imaging with a stereo-illuminated range-gated imaging system, in a watertight enclosure, as depicted in Fig. 7(b). High intensity LEDs (Light Emitting Diodes) forms an active illumination module that turns ON during the exposer period of cameras (in the PS_ON mode). A set of pulse-gated laser stripes are processed by a LSR module (the LSR_ON mode) which extends the optimal range of MARESye to retrieve the three-dimensional characteristic of underwater scenarios. This gating timing scheme makes it possible to create images with high signal-tonoise ratio.

\section{Results}

Overall, this section analyses the performance features of the proposed system and discusses the advantages comparatively to a conventional passive imaging system (CPIS). A comprehensive set of experiments were conducted as part of this work to assess how the perceptual losses caused by water turbidity affect on the visual response of MARESye. Therefore, the visual quality (in Section 4.2) and the capacity to retrieve the 3D information (in Section 4.3) are discussed according to several testing conditions, in particular, distinct objects and different concentration levels of suspended matter. Experiments were focused on the PS-module of MARESye since, theoretically, it is more affected by the water turbidity.

\footnotetext{
${ }^{5}$ The number of operating modes could be easily extended.
}

Table 1

Characterization of the testing conditions (trials): concentration level of matter and the illuminance of the external environment.

\begin{tabular}{lll}
\hline Turbidity level & Material Dissolved (mg/l) & Illuminance (lx) \\
\hline Baseline (clear water) & 0.0 & 95 \\
Low turbidity & 37.8 & 98 \\
Medium turbidity & 68.2 & 99 \\
High turbidity & 90.9 & 98 \\
\hline
\end{tabular}

First experiments provide a reliable study of photometric phenomena that affect underwater imaging (mainly, the backscattering component). They try to characterize the perceptual quality of MARESye and to compare the performance against a CPIS. A quantitative assessment is conducted using several objective metrics, namely, the root-mean square error (RMSE), the peak signal-to-noise ratio (PSNR) and the structural similarity (SSIM). The perceptual quality assessment is mainly obtained through the structural similarity (SSIM) metric since the SNR and PSNR have physical meanings but they are not suitable to perceive the visual quality [28]. Second experiments show the thee-dimensional reconstruction of objects as a function of the water turbidity. Quantitative evaluations are supported by the absolute mean error of point clouds generated by MARESye which are also compared to a conventional passive imaging system (CPIS). A total number of 30 point clouds from each testing condition make possible to specify the average error and standard deviation of measurements along coordinate axes (X, Y and Z). Several objects were considered during these trials. Finally, the third experiment aims to study the accuracy of MARESye, in particular, the LSR and the PS modules. This is particularly relevant for an optical system designed for precise and accurate underwater operations.

\subsection{The experimental setup}

Experiments have been conducted in a water tank $(0.94 \times 0.54 \times$ $0.52 \mathrm{~m}$ ) initially filled with clear water (264 litres), see Fig. 9(a) and (b). The turbidity of the water was controlled by dissolving different quantities of gray mortar to create distinct scattering conditions, similar to the ones that are usually found during close-range operations in real marine environments. The material remain suspended in water for a time sufficient to conduct the experiments. All results were obtained with a Secchi disk ${ }^{6}$ and by varying the concentration level of gray mortar dissolved in water as presented in Table 1: a baseline ( $0 \mathrm{mg} / \mathrm{l})$, low concentration $(37.8 \mathrm{mg} / \mathrm{l})$, medium concentration $(68.2 \mathrm{mg} / \mathrm{l})$ and high concentration $(90.9 \mathrm{mg} / \mathrm{l})$, were achieved by dissolving $0 \mathrm{~g}, 10 \mathrm{~g}, 18 \mathrm{~g}$ and $24 \mathrm{~g}$, respectively.

Moreover, all tests have been conducted with four clay objects ranging from 0.13 to $0.20 \mathrm{~m}$ : V-object, OS-object, OC-object and T-object, see Fig. 8(a)-(c).

The results are contextualized in an underwater environment to make possible a reliable and accurate evaluation of the proposed system. All results in this section were obtained with an i7-4700HQ CPU

\footnotetext{
6 The Secchi disk aims to facilitate the visual comparison between images acquired with distinct concentration levels of suspended matter.
} 


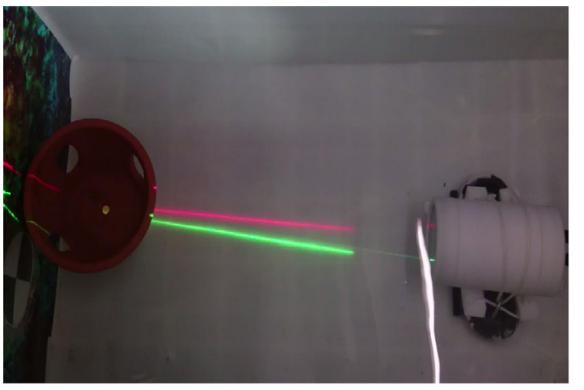

(a)

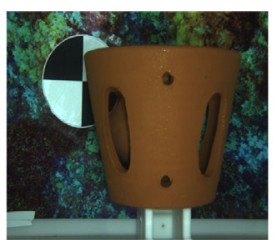

(a)

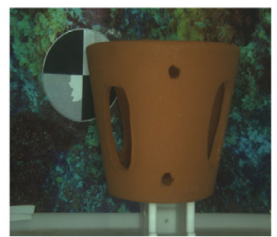

(e)

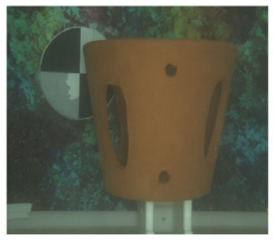

(i)

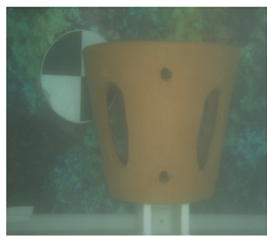

(m)

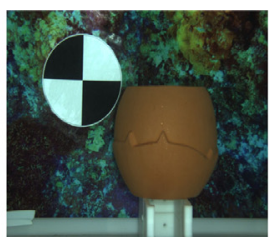

(b)

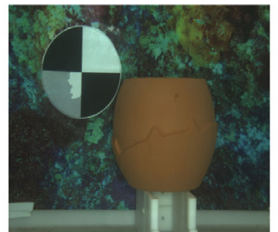

(f)

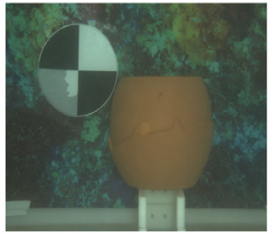

(j)

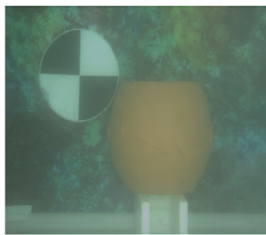

(n)

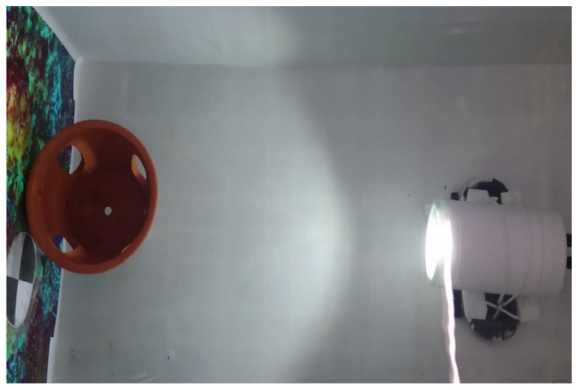

(b)

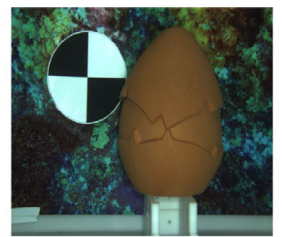

(c)

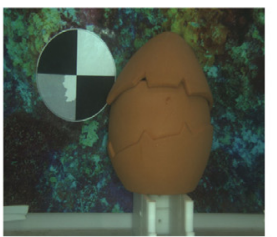

(g)

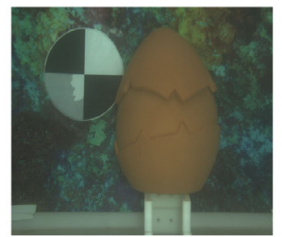

(k)

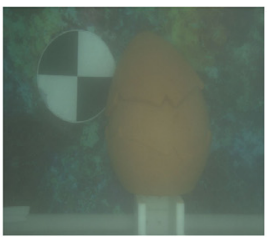

(o)

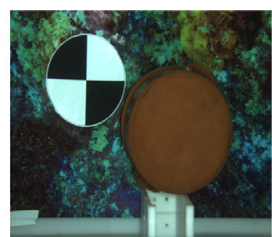

(d)

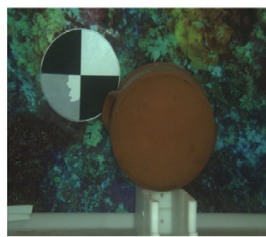

(h)

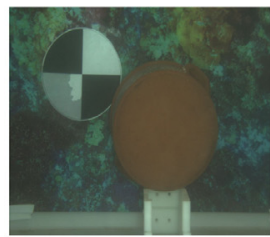

(1)

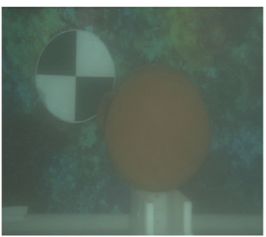

(p)
Fig. 9. Experimental setup during the acquisition of the V-object conducted by MARESye subjected to baseline conditions: LSR mode (on the left) and PS mode (on the right).

Fig. 10. The images (from the left-side) acquired by the MARESye (PS mode) during experiments characterized by clear water (Fig. 10(a)-(d)), low concentration (Fig. 10(e)-(h)), medium concentration (Fig. 10(i)-(1)) and high concentration (Fig. 10(m)-(p)) of suspended mater. All images were acquired under the same camera and gated-light settings: 3005 micro-seconds of exposure time and $0 \mathrm{~dB}$ of gain.
$2.40 \mathrm{GHz}$ computer and without parallel programing or GPU. The vision software was implemented in $\mathrm{C}++$ using the commonly used OpenCV library (version 3.1). Both the MARESye and the CPIS share a similar hardware. The CPIS relies on a "Lumen Subsea Light - BlueRobotics", physically separated by $0.2 m$ from the acquisition system. This setup follows imaging solutions that are usually found in ROV/AUVs. The images have a resolution of $1280 \times 960$ and are captured by a stereo-rig composed by two "MAKO 125C - AlliedVision" cameras with a $6 \mathrm{~mm}$ focal lens. The calibration of both visual systems was conducted in air and underwater following the global Levenberg-Marquardt optimization algorithm to minimize the re-projection error of points in a chessboard. Thus, the effectiveness of the calibration procedure was controlled by the re-projection error where in both cases was less than half of pixel. The refraction at the water-air interface causes a reduction of the field-of-view [3] because the imaging system hous- ing on Fig. 3 mounts a flat port. This phenomenon is depicted later on Figs. 12(a)-13(d).

\subsection{The perceptual quality}

The perceptual quality of the MARESye is evaluated in this section. Fig. 10(a)-(p) present four testing scenarios with different objects. These figures provide qualitative judgments of the visual performance of MARESye. The images captured by this system are affected by the concentration level of suspended matter since their contrast is reduced.

The quality of images acquired by MARESye and CPIS are compared during the trials/conditions presented in Table 1. The objective metrics, namely, RMSE, SNR, PSNR and SSIM quantify the degradation of the visual quality suffered by MARESye, according to distinct testing conditions (baseline, low, medium and high turbidity level). These metrics 


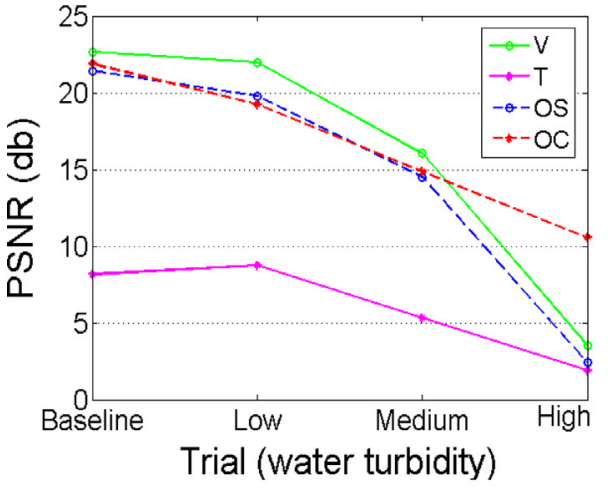

(a)

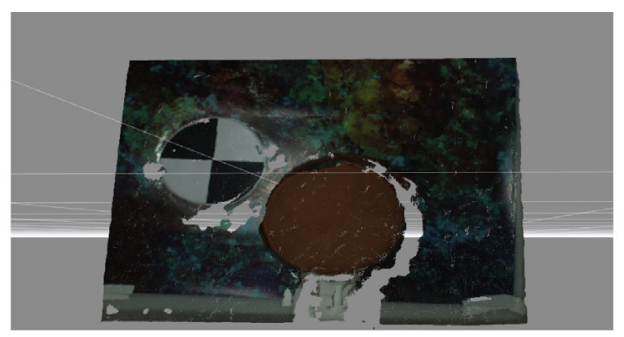

(a)

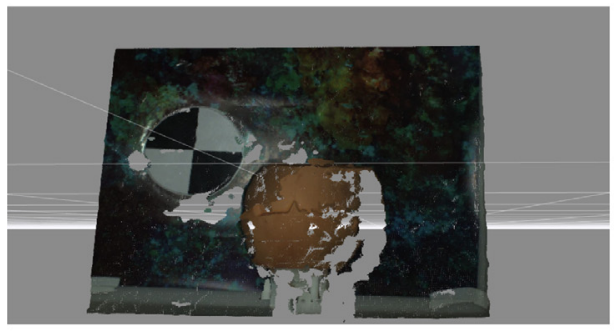

(c)

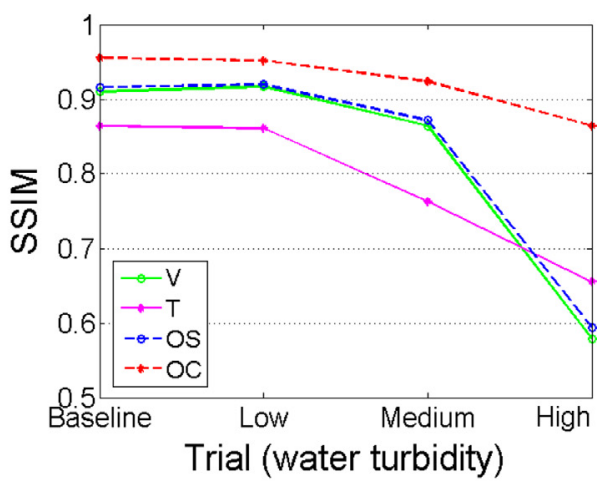

(b)

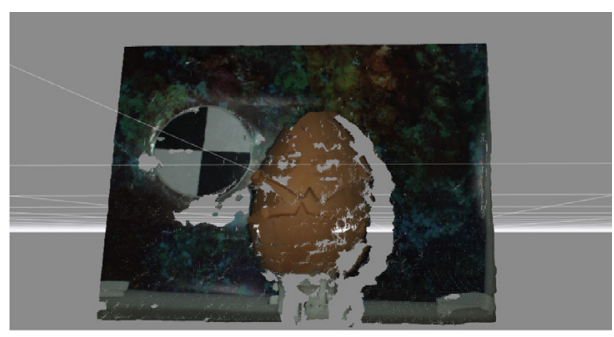

(b)

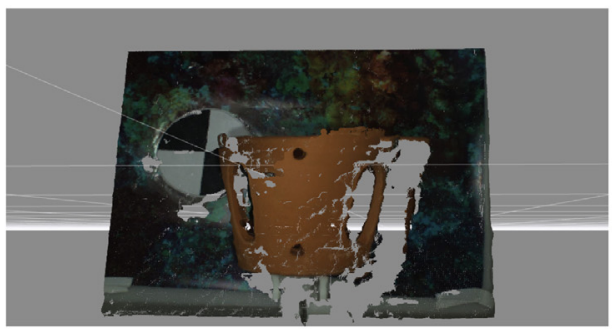

(d)
Fig. 11. Graphical representations of the performance evolution of MARESye system using the PSNR and SSIM assessment metrics for different turbidity conditions.
Fig. 12. 3D reconstruction of MARESye (PSmodule) during the aerial trial. are estimated relatively to aerial trials that were obtained for benchmarking. A region of interest formed only by the foreground surface of objects was specified for the determination of the RMSE, SNR, PSNR and SSIM values. Each region of interest was compared to the aerial trial. In this way, the visual losses caused by the underwater scenario can be established for different concentration levels of gray mortar, see Fig. 11(a) and (b) and Table 2. The results are focused on the PS-module because it is a passive imaging system and, therefore, is more easily affected by the water conditions, in particular, the reduction of the image contrast.

Fig. 11 (a) and(b) present the performance of MARESye for all testing conditions and considering the PSNR and SSIM metrics, respectively. The curves of both metrics follow a similar pattern whose differences are caused by the clarity of objects. This fact can be noticed by comparing the decay ratio of trials with the T-object versus the V-object: the visual quality of trials with T-object was degraded after the testing conditions with low turbidity. However, the visual quality of other objects was degraded after the medium turbidity trials. Therefore, images capturing darker objects (T-object) accelerate the perceptual quality loss since they cause images with lower contrast. The results of the MARESye system for all objects can be grouped in two sets: objects $\mathrm{OC}$ and $\mathrm{T}$ and, the objects V and OS; which means that, the perceptual quality obtained by the visual acquisition of objects belonging to each group share a similar profile. The shape of objects possibly affect the performance of this imaging system since regions of objects that are not properly illuminated can induce local shadows. The objects V and OS have more regular shapes that are uniformly illuminated when compared to objects T and OC, whose images have low contrast.

As expected, by increasing the concentration level of gray mortar the water turbidity is also increased which directly affects the quality of image. MARESye demonstrates a good response in terms of visual quality for baseline/medium turbidity trials since the maximum decay values were $7 \mathrm{~dB}$ for PNSR and 0.1 for SSIM. Only the high turbidity trials have originated a significant reduction of the image contrast and degraded texture information, causing a maximum decay value of $13 \mathrm{~dB}$ for PSNR and 0.28 for SSIM. Therefore, the concentrations of suspended material in water above $90.09 \mathrm{mg} / 1$ will certainly lead to images with poor SNR but, even in those trials, the perceptual quality of MARESye was up to $11 \mathrm{~dB}$ and 0.86 for PSNR and SSIM, respectively. These results could be justified by the range-gated approach combined with the artificial illumination module that make possible to obtain images with a good perceptual quality.

Table 2 compares the perceptual quality values obtained between the proposed system and CPIS, under the same testing conditions (Table 1). Results demonstrate that the MARESye and the CPIS have distinct evolutions when the water turbidity increases. Differences related to the visual quality between both systems can be observed: up to $10 \mathrm{~dB}$ and 0.5 for PSNR and SSIM, respectively. The images acquired by MARESye have demonstrated a higher perceptual quality (for T-object and OCobject), for instance, the SNR values of CPIS were reduced from 3.45 (T-object) and $16.80 \mathrm{~dB}$ (OC-object) to -12.68 (T-object) and $-7.11 \mathrm{~dB}$ 


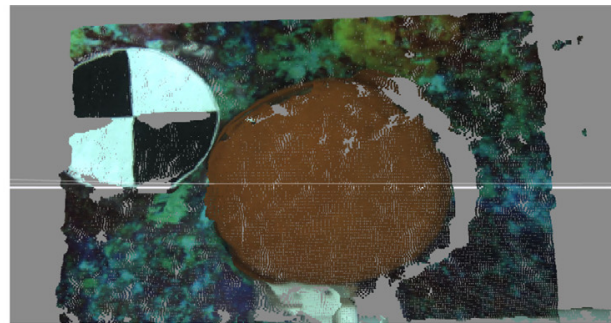

(a)

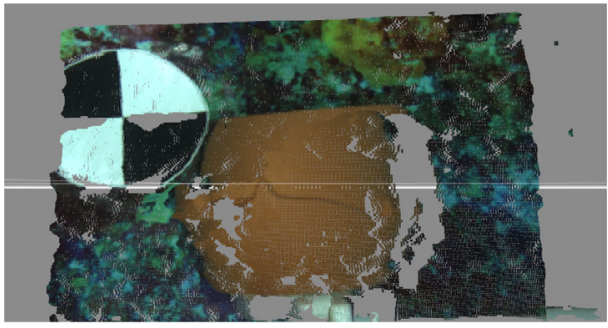

(c)

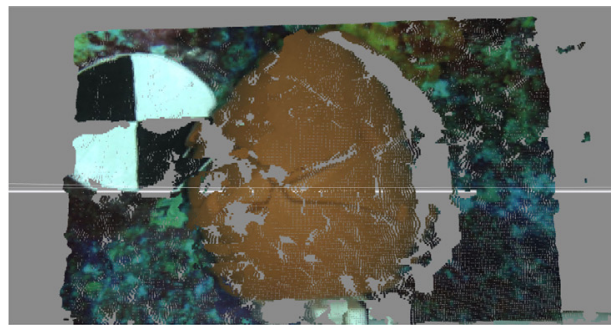

(b)

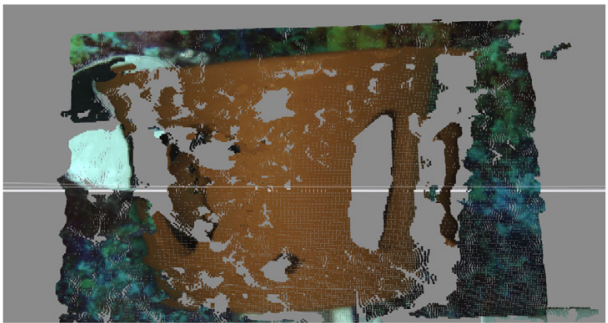

(d)
Fig. 13. 3D reconstruction of MARESye (PSmodule) during the baseline trial.
Table 2

Comparison between the MARESye (PS-module) and a CPIS, considering the RMSE, SNR, PSNR and SSIM assessment metrics for different turbidity conditions. The RMSE is represented in pixels and the SNR and PSNR are represented in decibels $(\mathrm{dB})$.

\begin{tabular}{llllll}
\hline Metric & T-object/System & Baseline & Low & Medium & High \\
\hline \multirow{2}{*}{ RMSE } & Conventional & 26.58 & 124.78 & 145.16 & 170.44 \\
& MARESye & - & $\mathbf{2 4 . 8 1}$ & $\mathbf{3 6 . 8 8}$ & $\mathbf{5 4 . 7 4}$ \\
SNR & Conventional & 3.45 & -9.97 & -11.28 & -12.68 \\
& MARESye & - & $\mathbf{4 . 0 5}$ & $\mathbf{0 . 6 1}$ & $\mathbf{- 2 . 8 1}$ \\
PSNR & Conventional & 8.15 & -5.27 & -6.58 & -7.98 \\
& MARESye & - & $\mathbf{8 . 7 5}$ & $\mathbf{5 . 3 1}$ & $\mathbf{1 . 8 8}$ \\
\multirow{2}{*}{ SSIM } & Conventional & 0.864 & 0.370 & 0.414 & 0.328 \\
& MARESye & - & $\mathbf{0 . 8 6 0}$ & $\mathbf{0 . 7 6 2}$ & $\mathbf{0 . 6 5 5}$ \\
\hline \multirow{2}{*}{ Metric } & OC-object/System & Baseline & Low & Medium & High \\
\hline \multirow{2}{*}{ RMSE } & Conventional & 9.57 & 131.13 & 136.37 & 150.38 \\
& MARESye & - & $\mathbf{1 3 . 0 1 5}$ & $\mathbf{2 1 . 4 6}$ & $\mathbf{3 5 . 2 2}$ \\
SNR & Conventional & 16.80 & -5.92 & -6.26 & -7.11 \\
& MARESye & - & $\mathbf{1 4 . 1 3}$ & $\mathbf{9 . 7 9}$ & $\mathbf{5 . 4 9}$ \\
\multirow{2}{*}{ PSNR } & Conventional & 21.89 & -0.84 & -1.18 & -2.03 \\
& MARESye & - & $\mathbf{1 9 . 2 2}$ & $\mathbf{1 4 . 8 7}$ & $\mathbf{1 0 . 5 7}$ \\
\multirow{2}{*}{ SSIM } & Conventional & 0.955 & 0.572 & 0.557 & 0.526 \\
& MARESye & - & $\mathbf{0 . 9 5 1}$ & $\mathbf{0 . 9 2 3}$ & $\mathbf{0 . 8 6 3}$ \\
\hline
\end{tabular}

(OC-object) while the values of MARESye (under the same conditions) were reduced to -2.81 (T-object) and $5.59 \mathrm{~dB}$ (OC-object). Moreover, the average SSIM difference of MARESye and CPIS was about 0.388 and 0.360 for T-object and OC-object.

Overall, the visual quality of MARESye is always higher than 0.6 for SSIM: from 0.95 to 0.87 in OC-object and 0.86 to 0.66 in T-object. Therefore, the PS-module of MARESye reduces the backscattering component when compared to a CPIS (that shares similar hardware setup and configuration).

\subsection{Three-dimensional reconstruction of underwater objects}

Results have demonstrated that the innovations presented in this paper enhances the perceptual quality of images acquired in underwater environments, even when the imaging system is operating with a high concentration level of suspended matter in water. The MARESye system has the ability to provide 3D data from its visual acquisitions and, therefore, this section goes one step further and evaluates the impact that such innovations have during the three-dimensional reconstruction of objects. Qualitative evaluations aim to evaluate the precision and accuracy of the proposed system (in particular, the PS module) are supported by quantitative metrics (absolute mean error) of the 3D reconstructions. In this article, the term "precision" relates to the statistical variability of data and the "accuracy" relates to the measurement error according to the ground truth.

\subsubsection{The precision of the $3 D$ data}

Extracting 3D information from 2D point pairs (known by stereoscopy) depends on the algorithm that is used to solve this association problem. In this research, the same algorithm [41] sharing an identical parametrization ${ }^{7}$ was used to obtain the following results.

Examples of the 3D reconstructions retrieved by the PS module of MARESye are represented in Figs. 12(a)-14(d). The point clouds for the four objects were obtained with different conditions: aerial are depicted in Fig. 12(a)-(d) to serve as a reference in this research (as indicated in Section 4.2), water with a low concentration of gray mortar is represented in Fig. 13(a)-(d) and, finally, the water with a medium concentration is represented in Fig. 14(a)-(d). As expected, the field-of-view of the MARESye diminishes from the trials in air to water since a flat port is required for the current housing however, this limitation can be solved in future upgrades. Two major results appear from the analysis of point clouds obtained during the medium trials, when compared to the ones obtained during the baseline (and, ultimately, to the aerial) trials: the precision and the density of points that are correctly estimated by MARESye are reduced by the increased the turbidity of water. High level contents of suspended matter augments the amount of light scattered in water which induces a noise component to the point clouds. This is mitigated but not completely eliminated by the imaging principles implemented in MARESye and, as a consequence, the algorithm that calculates the stereo correspondence had some difficulty during the trials with medium concentration levels, see Figs. 13(a)-14(a). Other example of this phenomenon can be seen in Figs. 13(c) and 14(c).

The precision of each system can be quantitatively studied using the absolute mean error and the standard deviation of measurements along each coordinate axis (X, Y and Z). A total number of 30 samples of point

\footnotetext{
7 Parametrization: size of the neighborhood (5), minimum disparity (4), number of disparities (80), penalties on the disparity change (2400 and 600), speckle range (2) and speckle window (10).
} 


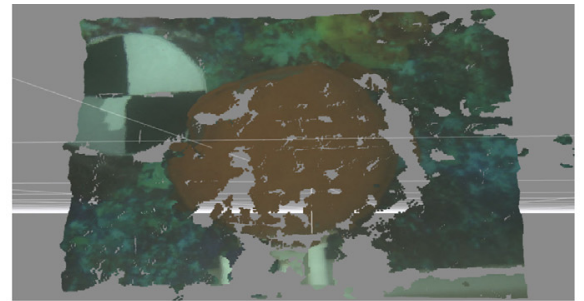

(a)

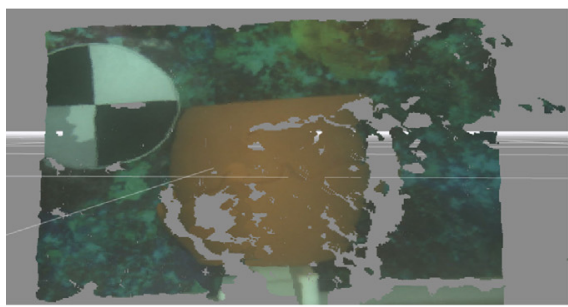

(c)

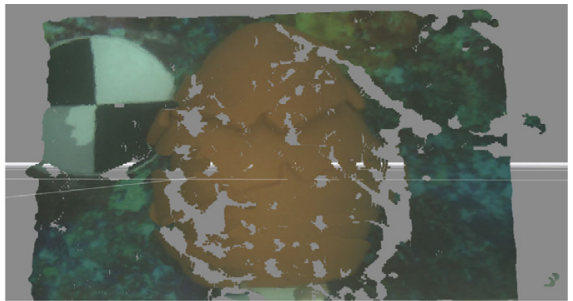

(b)

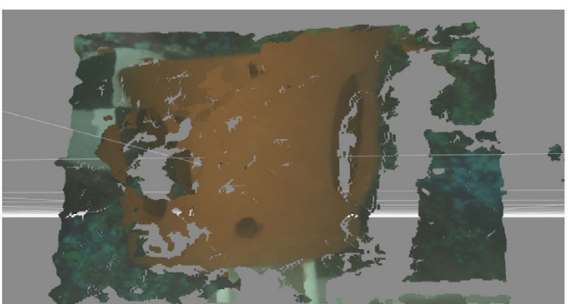

(d)
Fig. 14. 3D reconstruction of MARESye (PS-module) during the medium concentration trial.

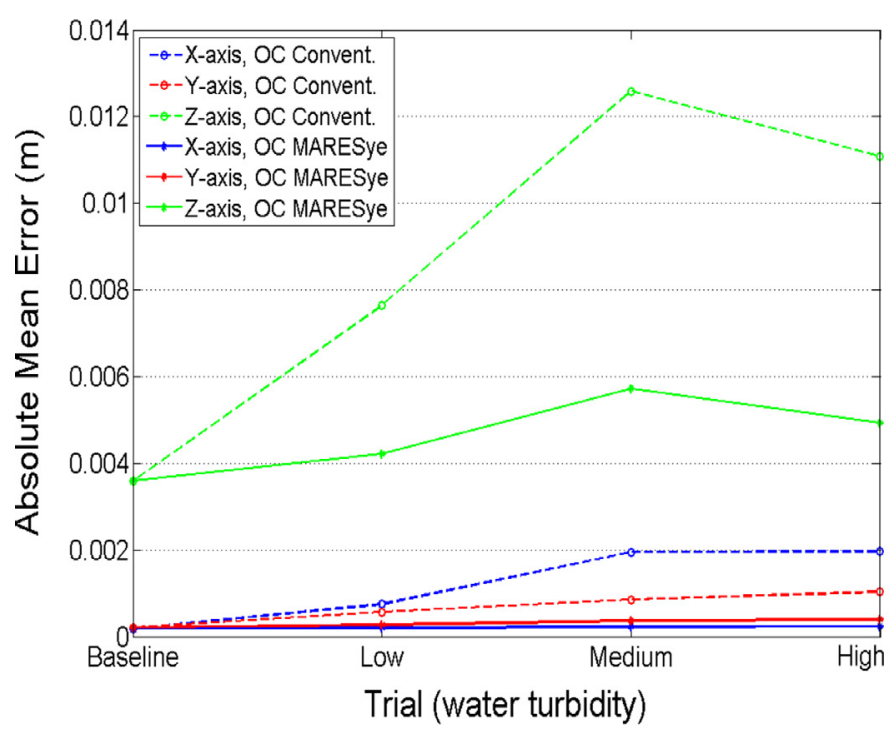

(a)

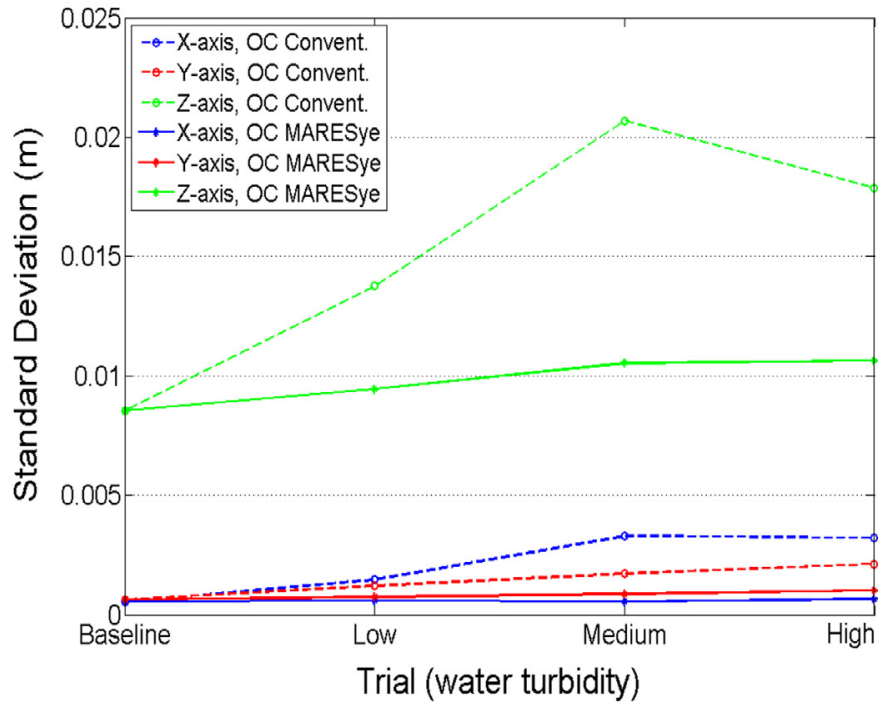

(b)

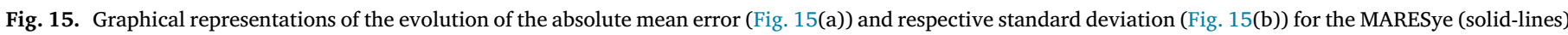
and the CPIS (dashed-lines), for different turbidity conditions. The results were obtained for the OC-object.

clouds were retrieved for each trial using the MARESye (PS module) and a CPIS. A reference can be estimated (a point cloud) using these 30 samples and after segmenting the object's surface that is visible on the water tank. Based on this reference, it is possible to estimate the absolute error of each 3D point, as well as, the average and standard deviation along each axis.

Figs. 15 (a)-16(a) represent the absolute mean error of the 3D surface of the OC-object and T-object, respectively. As can be noticed, the MARESye obtained lower errors. The maximum absolute mean error were lower than $0.001 \mathrm{~m}$ for $\mathrm{X}$ and $\mathrm{Y}$-axis and $0.006 \mathrm{~m}$ for Z-axis. On the other hand, the absolute mean errors of CPIS were close to 0.002, 0.001 and $0.013 \mathrm{~m}$ for the X, Y and Z-axis. Figs. 15(b) and 16(b) show the standard deviation of the absolute mean errors for both objects (OCobject and T-object). The PS-module of MARESye show a small standard deviation for the majority of axes, with the exception of the Zaxis of T-object where the values of both imaging systems were close to $0.015 \mathrm{~m}$ (for medium and high turbidity levels). Therefore, these results demonstrated an unambiguous enhancement of the precision of the PS-module of MARESye when compared to CPIS for operations involving the three-dimensional reconstruction of objects in underwater environments. In fact, it was proved that a range-gated imaging scheme reduces the impact of the backscattering component during the image formation and makes it possible to estimate point clouds with a better precision.

\subsubsection{The accuracy of the $3 D$ data}

This section evaluates the accuracy of MARESye for the PS and LSR modules, see Table 3. Results of the PS-module show measurements that were manually obtained through the point clouds of Figs. 12(a)-14(d). The real dimensions of all objects were compared to measures obtained by considering the Euclidean distance of $3 \mathrm{D}$ points that represent the height of each object. These results were also retrieved in different water conditions (from clear water to high turbidity). As can be noticed, the maximum absolute error was about $0.035 \mathrm{~m}$ and the average error was less than $0.025 \mathrm{~m}$. An important feature of this analysis is that, the accuracy does not appear to be affected by the water turbidity and thus, 


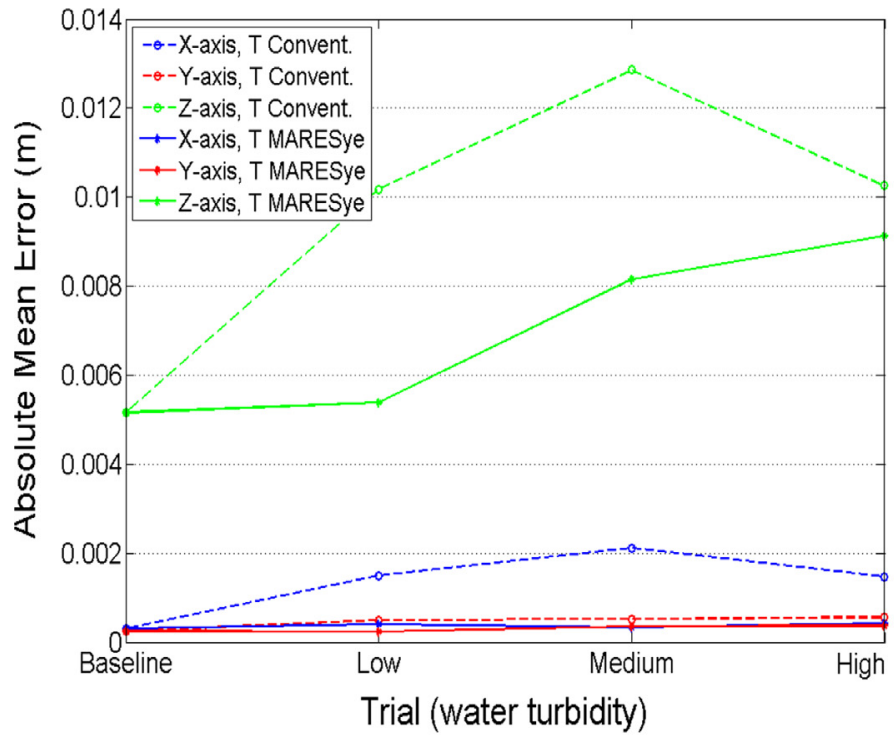

(a)

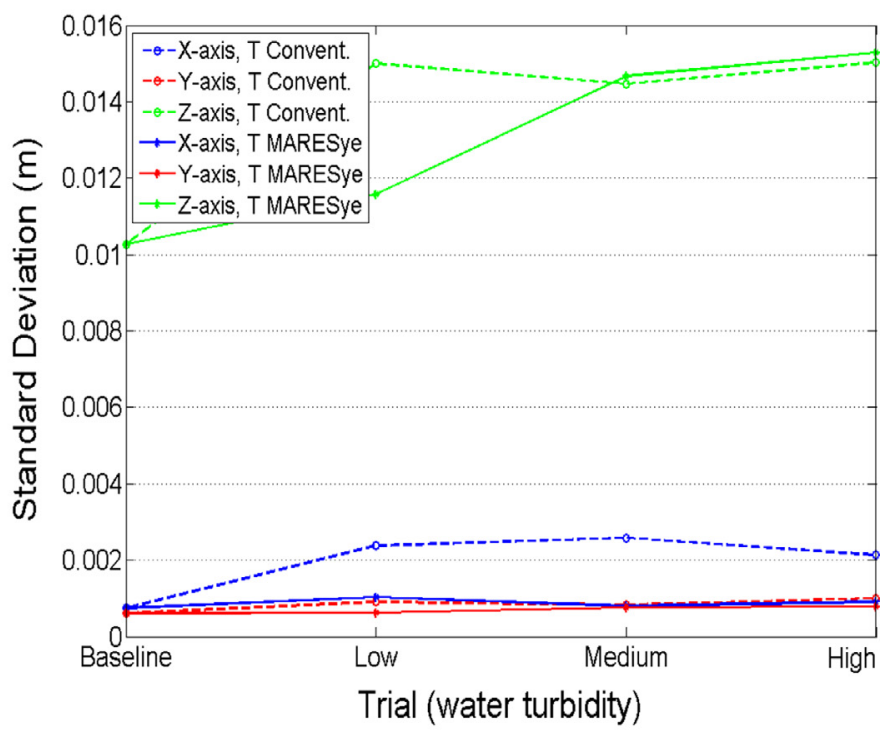

(b)

Fig. 16. Graphical representations of the evolution of the absolute mean error (Fig. 15(a)) and respective standard deviation (Fig. 15(b)) for the MARESye (solid-lines) and the CPIS (dashed-lines), for different turbidity conditions. The results were obtained for the T-object.

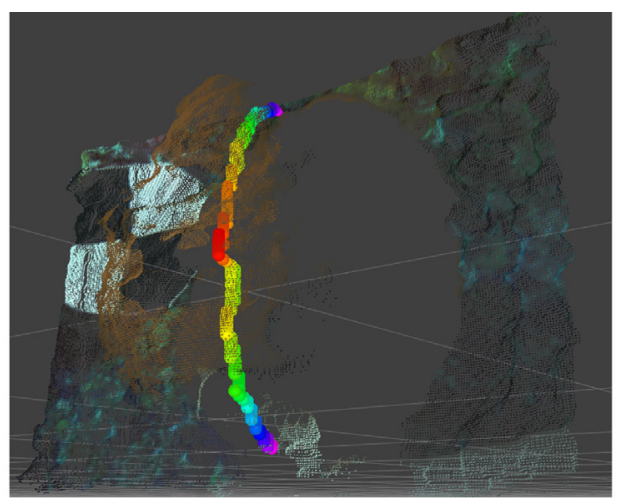

(a)

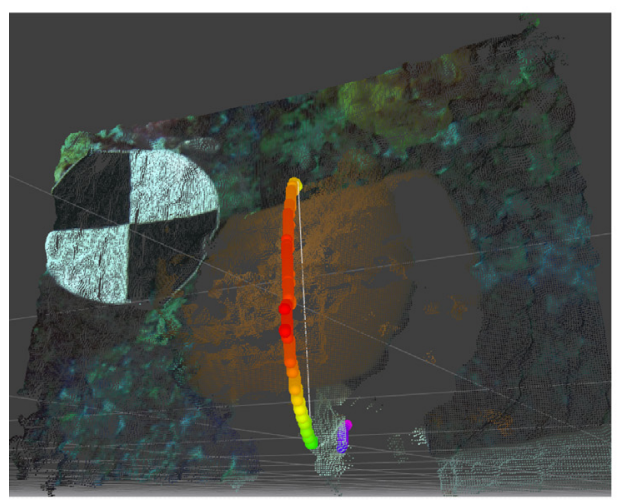

(c)

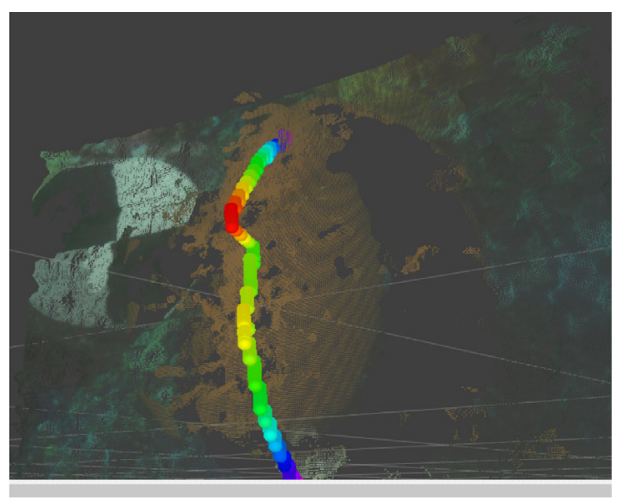

(b)

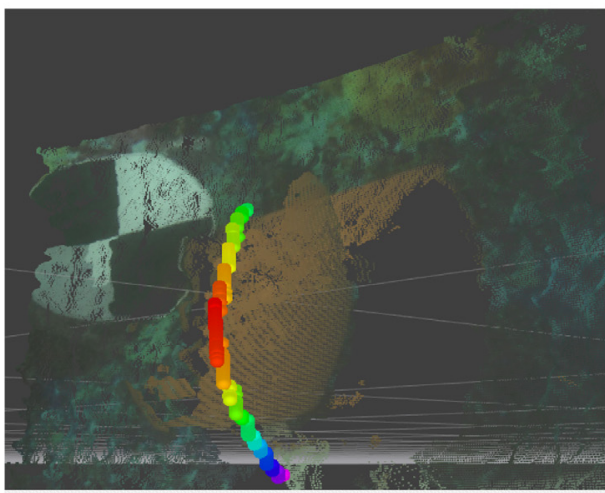

(d)
Fig. 17. 3D reconstruction of MARESye (LSRmodule) during the low (left column) and high (right column) turbidity trials. that the major impact of the turbidity on the precision of the 3D reconstructions.

The results of the LSR-module (a 50 milliwatt laser stripe with red wavelength) demonstrate that the maximum absolute error was round $0.020 \mathrm{~m}$ and the average absolute error was about $0.010 \mathrm{~m}$. Two major outcomes can be discussed from Table 3: measurements conducted using the LSR data are more accurate (lower error than the PS mod- ule) and this imaging technique is robust to underwater environment, see Fig. 17(a)-(d). These figures show the reconstruction of the 3D surface of OS-object and OC-object in the most extreme trials: baseline and high turbidity. As can be noticed, the 3D information retrieved by this module captures the surface of objects very accurately. Moreover, the 3D shape of objects are clearly depicted however, the density of points are limited to the laser stripe triangulation. Therefore, the hybrid sys- 
Table 3

Accuracy of MARESye (PS module) in different trials.

\begin{tabular}{llllll}
\hline Trial & Object & Measured by PS $(\mathrm{m})$ & Error of PS (m) & Measured by LSR (m) & Error of LSR (m) \\
\hline Baseline & T & 0.129 & 0.011 & 0.133 & 0.007 \\
& OC & 0.160 & 0.030 & 0.187 & 0.003 \\
& OS & 0.095 & 0.035 & 0.132 & 0.008 \\
Low & V & 0.143 & 0.017 & 0.176 & 0.016 \\
& T & 0.125 & 0.015 & 0.127 & 0.013 \\
& OC & 0.181 & 0.009 & 0.170 & 0.020 \\
Medium & OS & 0.104 & 0.026 & 0.132 & 0.008 \\
& V & 0.150 & 0.010 & 0.175 & 0.015 \\
& T & 0.122 & 0.018 & 0.128 & 0.012 \\
High & OC & 0.168 & 0.022 & 0.175 & 0.015 \\
& OS & 0.100 & 0.030 & 0.131 & 0.001 \\
& V & 0.139 & 0.001 & 0.172 & 0.012 \\
& T & 0.137 & 0.003 & 0.123 & 0.017 \\
& OC & 0.156 & 0.034 & 0.188 & 0.002 \\
& OS & 0.146 & 0.016 & 0.129 & 0.001 \\
& V & 0.159 & 0.019 & 0.169 & 0.009 \\
\hline
\end{tabular}

tem proposed in this research work comprises of two distinct imaging techniques, namely, the PS and LSR module that take the advantage of relevant features: density, robustness and accuracy of 3D points. Formulating both modules in a range-gated imaging scheme with a data-driven principle makes it possible to create different sources of data that can be used to support several tasks, for instance, for underwater navigation of mobile robots, recognition and manipulation of objects.

\section{Conclusion}

This research presented a novel imaging system suitable for underwater scenarios. This hybrid system is called MARESye and accommodates several features that mitigate the usual photometric limitations of conventional underwater imaging systems. MARESye is formed by two major components, namely, a multiple light stripe range (LSR) and a photometric stereo (PS) unit. Unlike other conventional imaging systems, the proposed solution relies on a hybrid approach controlled by a range-gated imaging scheme and a data-driven formulation.

Extensive experiments have quantified the visual quality and the accuracy of the three-dimensional information that can be retrieved by the proposed system. These experiments depict realistic sub-sea conditions and prove that concepts explored in MARESye have the ability to enhance the quality of the visual information. In fact, the range-gated scheme combined with a data-driven formulation makes possible to explore the advantages of both passive and active techniques, as well as, to mitigate the imaging effects related to the light propagation in deep waters. In practice, the perceptual quality of MARESye achieved a SSIM index above 0.655 for waters having a high concentration of suspended material while, at the same time, a conventional system obtained a value of 0.328. In addition, the results also demonstrate that MARESye reconstructs the 3D surface of objects with a high accuracy and precision, since the average Euclidean error was lower than $0.025 \mathrm{~m}$ and $0.010 \mathrm{~m}$ for the PS and LSR modules, respectively.

Therefore, MARESye is a hybrid imaging system specially developed for retrieving visual information and to conduct 3D underwater reconstructions. The robustness level of the information perceived by the system under different environmental conditions makes the MARESye suitable for autonomous or remotely-operated vehicles.

\section{Acknowledgment}

This work is financed by the ERDF European Regional Development Fund through the Operational Programme for Competitiveness and Internationalisation - COMPETE 2020 Programme and by National Funds through the Portuguese funding agency, FCT - Fundacão para a Ciência e a Tecnologia within project DIIUS -Distributed perceptIon for inspec-
tIon of aqUatic Structures - POCI-01-0145-FEDER-030010. The authors also would like to acknowledge Nuno Alexandre Cruz for the design of the watertight enclosure of MARESye.

\section{References}

[1] F. Bonin-Font, A. Burguera, G. Oliver, Imaging systems for advanced underwater vehicles, J. Marit. Res. 8 (1) (2011) 65-86.

[2] F. Bonin-Font, G. Oliver, S. Wirth, M. Massot, P. luis Negre, J.-P. Beltran, Visual sensing for autonomous underwater exploration and intervention tasks, Ocean Eng. 93 (2015) 25-44, doi:10.1016/j.oceaneng.2014.11.005.

[3] F. Bruno, G. Bianco, M. Muzzupappa, S. Barone, A. Razionale, Experimentation of structured light and stereo vision for underwater 3D reconstruction, ISPRS J. Photogramm. Remote Sens. 66 (4) (2011) 508-518, doi:10.1016/j.isprsiprs.2011.02.009.

[4] P. Carrasco, F. Bonin-Font, M. Campos, G. Codina, Stereo-vision graph-slam for robust navigation of the AUV SPARUS II, IFAC-PapersOnLine 48 (2) (2015) 200-205, doi:10.1016/j.ifacol.2015.06.033. 4th IFAC Workshop onNavigation, Guidance and Controlof Underwater VehiclesNGCUV 2015

[5] F. Dalgleish, A. Vuorenkosk, B. Ouyang, Extended-range undersea laser imaging: current research status and a glimpse at future technologies, Mar. Technol. Soc. J. 47 (5) (2013) 128-147.

[6] H. Duo-Min, Underwater laser-illuminated range-gated imaging scaled by $22.5 \mathrm{~cm} \mathrm{~ns}^{-1}$ with serial targets, J. Ocean Univ. China 3 (2) (2004) 208-219, doi:10.1007/s11802-004-0037-4.

[7] B. Elnashef, S. Filin, Direct linear and refraction-invariant pose estimation and calibration model for underwater imaging, ISPRS J. Photogramm. Remote Sens. 154 (2019) 259-271, doi:10.1016/j.isprsjprs.2019.06.004.

[8] M.S. Farid, A. Mahmood, S.A. Al-Maadeed, Multi-focus image fusion using content adaptive blurring, Inf. Fusion 45 (2019) 96-112, doi:10.1016/j.inffus.2018.01.009.

[9] P. Felzenszwalb, D. Huttenlocher, Efficient belief propagation for early vision, Int. J. Comput. Vis. 70 (1) (2006) 1-12.

[10] N. Gracias, J. Santos-Victor, Underwater video mosaics as visual navigation maps, Comput. Vis. Image Underst. 79 (1) (2000) 66-91, doi:10.1006/cviu.2000.0848.

[11] M. Hammoudeh, R. Newman, Information extraction from sensor networks using the watershed transform algorithm, Inf. Fusion 22 (2015) 39-49, doi:10.1016/j.inffus.2013.07.001.

[12] T. Hanning, A. Lasaruk, T. Tatschke, Calibration and low-level data fusion algorithms for a parallel 2D/3D-camera, Inf. Fusion 12 (1) (2011) 37-47, doi:10.1016/j.inffus.2010.01.006. Special Issue on Intelligent Transportation Systems

[13] N. Hansen, M.C. Nielsen, D.J. Christensen, M. Blanke, Short-range sensor for underwater robot navigation using line-lasers and vision, IFAC-PapersOnLine 48 (16) (2015) 113-120, doi:10.1016/j.ifacol.2015.10.267. 10th IFAC Conference on Manoeuvring and Control of Marine Craft MCMC 2015

[14] H. Hirschmuller, Efficient belief propagation for early vision, IEEE Trans. Pattern Anal. Mach. Intell. 30 (2) (2008) 328-341.

[15] J. Jaffe, Computer modeling and the design of optimal underwater imaging systems, IEEE J. Ocean. Eng. 15 (1) (1990) 101-111.

[16] J.S. Jaffe, Underwater optical imaging: the past, the present, and the prospects, IEEE J. Ocean. Eng. 40 (3) (2015) 683-700, doi:10.1109/JOE.2014.2350751.

[17] R. Kawahara, S. Nobuhara, T. Matsuyama, Dynamic 3D capture of swimming fish by underwater active stereo, Methods Oceanogr. 17 (2016) 118137, doi:10.1016/j.mio.2016.08.002. Special section on Novel instrumentation in Oceanography: a dedication to Rob Pinkel

[18] M. Ko, G. West, S. Venkatesh, M. Kumar, Using dynamic time warping for on line temporal fusion in multisensor systems, Inf. Fusion 9 (3) (2008) 370-388, doi:10.1016/j.inffus.2006.08.002. Special Issue on Distributed Sensor Networks 
[19] T. Letessier, J.-B. Juhel, L. Vigliola, J. Meeuwig, Low-cost small action cameras in stereo generates accurate underwater measurements of fish, J. Exp. Mar. Biol. Ecol. 466 (2015) 120-126, doi:10.1016/j.jembe.2015.02.013.

[20] J. Li, J. Besada, A. Bernardos, P. Tarrio, J. Casar, A novel system for object pose estimation using fused vision and inertial data, Inf. Fusion 33 (2017) 15-28, doi:10.1016/j.inffus.2016.04.006

[21] J. Li, M. Lee, W. Lee, J. Kim, H. Kang, J. Suh, Real time obstacle detection in a water tank environment and its experimental study, in: IEEE (Ed.), Proceedings of the IEEE/OES Autonomous Underwater Vehicles (AUV), 2014, pp. 1-5.

[22] J. Li, Y. Li, Underwater image restoration algorithm for freeascending deep-sea tripods, Opt. Laser Technol. 110 (2019) 129-134, doi:10.1016/j.optlastec.2018.05.034. Special Issue: Optical Imaging for Extreme Environment

[23] S. Li, X. Kang, L. Fang, J. Hu, H. Yin, Pixel-level image fusion: a survey of the state of the art, Inf. Fusion 33 (2017) 100-112, doi:10.1016/j.inffus.2016.05.004.

[24] Y. Liu, X. Chen, Z. Wang, Z. Wang, R. Ward, X. Wang, Deep learning for pixel-level image fusion: recent advances and future prospects, Inf. Fusion 42 (2018) 158-173, doi:10.1016/j.inffus.2017.10.007.

[25] J. Lu, N. Li, S. Zhang, Z. Yu, H. Zheng, B. Zheng, Multi-scale adversarial network for underwater image restoration, Opt. Laser Technol. 110 (2019) 105-113, doi:10.1016/j.optlastec.2018.05.048. Special Issue: Optical Imaging for Extreme Environment

[26] H. Madjidi, S. Negahdaripour, On robustness and localization accuracy of optical flow computation for underwater color images, Comput. Vis. Image Underst. 104 (1) (2006) 61-76, doi:10.1016/j.cviu.2006.07.003.

[27] F. Maurelli, J. Cartwright, N. Johnson, Y. Petillot, Herriot Watt oceans systems lab Nessie IV autonomous underwater vehicle wins the sauce competition, in: IEEE (Ed.), Proceedings of the IEEE International Conference on Autonomous Robot Systems and Competitions (ICARSC), 2010, pp. 87-93.

[28] A. Maykol, P. Costa, M. Velhote, A. Moreira, Enhancing dynamic videos for surveillance and robotic applications: the robust bilateral and temporal filter, Signal Process. Image Commun. 29 (1) (2014) 80-95, doi:10.1016/j.image.2013.11.003.

[29] F. Oleari, F. Kallasi, D.L. Rizzini, J. Aleotti, S. Caselli, An underwater stereo vision system: from design to deployment and dataset acquisition, Genova, in: Proceedings of the OCEANS, 2015, pp. 1-6, doi:10.1109/OCEANS-Genova.2015.7271529.

[30] M. Oliveira, V. Santos, A.D. Sappa, Multimodal inverse perspective mapping, Inf. Fusion 24 (2015) 108-121, doi:10.1016/j.inffus.2014.09.003.

[31] A. Pinto, M. Correia, A. Moreira, P. Costa, Unsupervised flow-based motion analysis for an autonomous moving system, Image Vis. Comput. 32 (6-7) (2014) 391-404.
[32] A. Pinto, P. Costa, M. Correia, A. Matos, A. Moreira, Visual motion perception for mobile robots through dense optical flow fields, Robot. Auton. Syst. 87 (2017) 1-14, doi:10.1016/j.robot.2016.08.014.

[33] A. Pinto, A. Moreira, P. Costa, WirelessSyncroVision: wireless synchronization for industrial stereoscopic systems, Int. J. Adv. Manuf. Technol. 82 (5) (2016) 909-919.

[34] A. Pinto, A. Moreira, M. Velhote, P. Costa, A flow-based motion perception technique for an autonomous robot system, J. Intell. Robot. Syst. 75 (3-4) (2013) 475-492, doi:10.1007/s10846-013-9999-z.

[35] A. Pinto, E. Moreira, J. Lima, J. Sousa, P. Costa, A cable-driven robot for architectural constructions: a visual-guided approach for motion control and path-planning, Auton. Robot. (2016), doi:10.1007/s10514-016-9609-6.

[36] A. Pinto, H. Pinto, A.C. Matos, A mosaicking approach for visual mapping of large-scale environments, in: IEEE (Ed.), Proceedings of the IEEE International Conference on Autonomous Robot Systems and Competitions (ICARSC), 2016, pp. 87-93.

[37] D. Ribas, N. Palomeras, P. Ridao, M. Carreras, A. Mallios, Girona 500 AUV: from survey to intervention, IEEE/ASME Tran. Mechatron. 1 (2012) 4653.

[38] D.L. Rizzini, F. Kallasi, J. Aleotti, F. Oleari, S. Caselli, Integration of a stereo vision system into an autonomous underwater vehicle for pipe manipulation tasks, Comput. Electr. Eng. 58 (1) (2017) 560-571, doi:10.1016/j.compeleceng.2016.08.023.

[39] A. Sarafraz, B.K. Haus, A structured light method for underwater surface reconstruction, ISPRS J. Photogramm. Remote Sens. 114 (2016) 40-52.

[40] R. Schettini, S. Corchs, Underwater image processing: state of the art of restoration and image enhancement methods, EURASIP J. Adv. Signal Process. 2010 (1) (2010) 746052.

[41] W. van der Mark, D.M. Gavrila, Real-time dense stereo for intelligent vehicles, IEEE Trans. Intell. Transp. Syst. 7 (1) (2006) 38-50.

[42] K. Williams, A.D. Robertis, Z. Berkowitz, C. Rooper, R. Towler, An underwater stereo-camera trap, Methods Oceanogr. 11 (2014) 1-12, doi:10.1016/j.mio.2015.01.003.

[43] A. Yang, L. Wang, N. Ahuja, A constant-space belief propagation algorithm for stereo matching, in: Proceedings of the International Conference on Computer Vision and Patter Recognition, 2010, pp. 1-6.

[44] H. Zhang, S. Negahdaripour, Epiflowa paradigm for tracking stereo correspondences, Comput. Vis. Image Underst. 111 (3) (2008) 307-328, doi:10.1016/j.cviu.2008.01.001.

[45] Q. Zhang, Y. Wang, M.D. Levine, X. Yuan, L. Wang, Multisensor video fusion based on higher order singular value decomposition, Inf. Fusion 24 (2015) 54-71, doi:10.1016/j.inffus.2014.09.008. 